\title{
MASSVA: Modelo de Avaliação de Sistemas Sociais sob a ótica dos Valores humanos nas postagens dos usuários
}

\author{
Denilson C Oliveira ${ }^{1}$, Elizabeth S. Furtado ${ }^{1}$, Marilia S. Mendes ${ }^{2}$ \\ ${ }^{1}$ PPGIA. Universidade de Fortaleza (Unifor), Fortaleza, CE - Brasil. \\ ${ }^{2}$ Universidade Federal do Ceará (UFC), Russas, CE - Brasil. \\ \{denilsoncursinol, elizabethsfur, mariliamendes\}@gmail.com
}

\begin{abstract}
Social Systems (SS) allow diverse interactive experiences to their users, whose posts, in natural language, are being exploited for various purposes. This paper proposes a model for the evaluation of an SS through the investigation of expressed human values of the users' posts. Twitter data were analyzed, according to the concepts of the model, with the purpose of verifying the utility of such values for the user interaction with these SS. The results pointed to the identification of quality problems in use of this SS, as well as to the possibility of improvements through the discovery of relationships between personal and social values, expressed in the analyzed posts.
\end{abstract}

Resumo. Sistemas Sociais (SS) possibilitam experiências interativas diversas aos seus usuários, cujas postagens em linguagem natural vêm sendo exploradas para diversos fins. Este artigo propõe um modelo para a avaliação de um SS a partir da investigação de valores humanos expressos das postagens dos usuários. Dados do Twitter foram analisados, segundo os conceitos do modelo, com o propósito de verificar a utilidade de tais valores para a interação do usuário com esses SS. Os resultados apontaram para a identificação de problemas de qualidade em uso desse SS, bem como para a possibilidade de melhorias a partir da descoberta de relacionamentos entre valores pessoais e sociais, expressos nas postagens analisadas.

\section{Introdução}

Diferentes usuários de SS interagem entre si, produzindo informações (postagens de usuários) úteis para aferir as medidas da qualidade em uso com o referido sistema, como: de usabilidade e da experiência do usuário (User eXperience, UX). O uso de postagens para avaliar a UX em SS permite identificar as conversas dos usuários, suas dúvidas, buscas por novos conhecimentos, entre outros objetivos que levam os usuários a interagirem nos SS (Mendes, 2015). Pereira et al. (2010) ressalta a importância em analisar os valores pessoais (p. ex., autonomia, privacidade, emoção e afeto, etc.) e sociais (p. ex., colaboração, compartilhamento, propriedade, etc.) existentes em um SS para avaliar os valores técnicos de tal sistema (aqui sendo considerados as medidas de 
qualidade em uso). Nenhum desses autores, nem de outros trabalhos apresentados na seção de trabalhos relacionados, focam na análise da expressão de valores nas postagens em linguagem natural e realizam o mapeamento da interação (como das medidas de qualidade em uso) com os valores pessoais e entre estes com os valores sociais. Quando nos referirmos à análise da expressão de Valores nas postagens feitas durante uma UX com o SS sendo avaliado, a abreviação VUX será usada. Em Oliveira et al. (2016), os autores deste artigo em submissão mostraram que as postagens dos usuários podem expressar valores pessoais e sociais, e ser uma fonte de conhecimento para auxiliar na avaliação da qualidade em uso de SS. Apesar disto, eles não mostraram: quais valores pessoais e sociais são mais recorrentes e como estes podem ser úteis para avaliar a VUX dos SS? O objetivo deste artigo é investigar as postagens dos usuários de um SS, do ponto de vista da VUX, com os propósitos de explorar os relacionamentos entre valores e analisar suas implicações para a qualidade em uso desse sistema. Esta pesquisa avançou para a definição de um modelo de dados de avaliação de um SS, representando os conceitos (e suas relações) usados para uma análise VUX. Esse modelo, chamado MASSVA (Modelo de Avaliação de SS sob a ótica de Valores), foi usado para guiar a análise e classificação de valores feitas com três extrações de postagens do Twitter, totalizando 5.326 postagens analisadas. Os resultados apontaram para a identificação de problemas de qualidade em uso do SS e possibilidades de melhorias do seu conteúdo e interface.

Este artigo está organizado da seguinte forma: a seção 2 apresenta fundamentações teóricas antes da apresentação do MASSVA; o modelo de dados MASSVA é apresentado na seção 3; na seção 4 ocorre a aplicação do modelo em um contexto específico de avaliação; os resultados são apresentados na seção 5; em 6, são apresentados os artigos do estado da arte relacionados à avaliação de SS usando valores; uma discussão sobre a aplicação do modelo proposto para analisar a VUX é feita na seção 7 e, por fim, tem-se a conclusão.

\section{Fundamentação Teórica}

\subsection{Sistemas Sociais e suas postagens}

Uma das primeiras definições sobre o termo Sistema Social foi descrita por Shirky (2005), onde SS são descritos como softwares que permitem interações em grupo. McLoughlin e Lee (2007) foram além, afirmando que os SS não possuem somente a possibilidade de interação entre seus usuários, mas têm a capacidade de recombinar e transformar todas as informações compartilhadas e seus próprios meios de transmissão em novas ferramentas. Como mencionado, eles vêm sendo explorados para diversos fins: i) educativos; Klamma et al. (2011) direcionam os SS para o contexto das tecnologias educativas e os definem como ferramentas que disponibilizam atividades educativas em redes sociais; ii) segurança; Bolea (2015) fez uma análise da carga de sentimentos e do vocabulário utilizado em postagens identificando um sentimento de perigo sobre os fatos que ocorreram ou iriam ocorrer para analisar a segurança. Em Beiming (2014) foi realizada uma análise da influência dos sentimentos nas postagens como um fator preponderante de atenção dos outros usuários em interagir com uma referida postagem. Um estudo sobre a possibilidade da existência de links maliciosos e spans nas postagens do Facebook, é realizado por Abu-Nimeh et al. (2011); e iii) avaliação da interação: Pereira et al. (2010) aprofundaram os conceitos sobre os SS, 
analisando seus valores sob os preceitos apresentados por Morville (2004): a colmeia de UX, que contém como medidas da UX, a usabilidade, privacidade, acessibilidade, etc. Já Smith (2008) utilizou estas medidas, dentre outros elementos para apresentar um Framework conceitual para avaliar SS, sendo eles: presença, compartilhamento, conversação, identidade, grupo, reputação e relacionamento.

Este trabalho se situa neste último propósito, e estende a proposta apresentada por Mendes (2015). A autora propôs um modelo, chamado MALTU, para avaliar a interação em SS a partir de Postagens Relacionadas ao Uso (PRUs). Uma PRU é uma postagem na qual seu conteúdo diz respeito a algo relacionado ao uso do sistema (p. ex., "Meu twitter tá com problema."). Entretanto, a autora descarta o uso de Não-PRU, ou seja, uma Postagem Não Relacionada ao uso do sistema, com conteúdo relacionado a qualquer assunto (p. ex., "Adorei o episódio de Breaking Bad de ontem"). Neste trabalho, para a análise VUX, os dois tipos de postagens serão usados.

\subsection{Valores Humanos (pessoais e sociais) e sua associação a PRU e Não-PRU}

Pereira et al. (2010) fazem a divisão dos valores em pessoais e sociais, os quais são utilizados nesta pesquisa. Um valor social refere-se aos impactos econômicos, sociais e ambientais gerados por uma atividade. Enquanto o pessoal é o conjunto de características de uma determinada pessoa ou organização, que determinam a forma como a pessoa ou organização se comportam e interagem com outros indivíduos e com o meio ambiente. Nos parágrafos que se seguem, o conceito de cada valor será brevemente descrito.

Os valores pessoais, apresentados em Pereira et al. (2010), são os seguintes:

- Autonomia: a origem etimológica desta palavra vem do termo grego "autos nomos" que implica em: autodeterminação, próprias regras. Holanda (2010) apresenta três significados básicos: faculdade que conserva um país conquistado de se administrar por suas próprias leis, liberdade moral ou intelectual e independência administrativa;

- Confiança: sentimento de quem confia, de quem acredita na sinceridade de algo ou de alguém (Holanda, 2010). Mayer (1995) afirma que a confiança se faz necessária onde há riscos, incertezas e interdependência;

- Consentimento Informado (CI): este valor teve sua importância ressaltada inicialmente por Belmont Report (1978), que discute sobre a construção de princípios éticos e diretrizes a serem abordados no desenvolvimento do consentimento informado durante as interações humanas no mundo digital;

- Emoção e Afeto: o interesse filosófico pelas emoções de meados do século XX pode ser atribuído a Bedford (1957) e Kenny (1963) por terem argumentado sobre a suposição de que as emoções são sentimentos impermeáveis a qualquer um;

- Identidade: segundo Hogg et al. (1988), identidade é o conceito das pessoas de quem elas são, de que tipo de pessoas elas são e como elas se relacionam com os outros;

- Presença: Garau (2003) apresenta três conceitos de presença: presença, presença social e co-presença, e define que presença é a sensação de "estar lá/ 
imersão" (p. ex., entretenimento, cinema, etc.), presença social como "estar junto" (p. ex., vídeo-chamada, videoconferência, etc.) e co-presença como "estar junto em um ambiente compartilhado" (p. ex., e-mail, telefone, chat, etc.);

- Privacidade: diz respeito à intimidade de pessoal ou de grupo definido de pessoas (Holanda, 2010). Ou como o valor que nos concede a capacidade de controlar o acesso que os outros têm a nós (Schoeman, 1992).

- Reciprocidade: para Sabourin (2008), é a obrigação de dar, receber e retribuir.

- Reputação: diz respeito à opinião pública, sendo esta favorável ou não a respeito à pessoa física ou jurídica (Holanda, 2010);

- Visibilidade: diz respeito ao fato do indivíduo ser encontrado, visto em um determinado local.

Adicionamos outros valores pessoais ao conjunto de valores que ajudarão a analisar a VUX de um SS, são eles:

- Conhecimento: Japiassu (1977) afirma "É considerado saber, um conjunto de conhecimentos metodicamente adquiridos, mais ou menos sistematicamente organizados, susceptíveis de serem transmitidos por um processo pedagógico de ensino";

- Responsabilidade Social: pode ser considerada uma ciência onde a busca de conhecimento para o bem-estar da sociedade deve ser aplicada por profissionais de diversas áreas (Arevalo, 2010);

- Religiosidade: é o sentimento humano que impele a reconhecer a divindade independentemente de culto determinado (Holanda, 2010);

- Prestígio: Huang (2009) divide o valor de prestígio em virtual, social e estrutural: o primeiro é medido pela quantidade de visitas recebidas no perfil de um determinado usuário; o segundo é percebido não pelo número de visitas e sim pelo forte sentimento de aliança e respeito por parte dos visitantes do perfil do usuário e o último é percebido a partir da análise de diversas variáveis para poder determiná-lo.

Os valores humanos, apontados como sociais em Pereira (2010) são utilizados nesta pesquisa. São eles:

- Normas, Regras e Políticas (NRP): segundo Dron (2007), normas, regras e políticas fornecem o princípio da confiança, sendo necessárias para que se possa construir mecanismos confiáveis de interação entre as pessoas e recursos sob a regência do valor NRP;

- Colaboração: diz respeito a agir com outrem para a obtenção de determinado resultado (Holanda, 2010). Tsai (2008) trabalha o conceito de colaboração sobre vários aspectos: a colaboração servindo como alicerce para a criação de comunidades online de aprendizado; e que a natureza social da educação depende da interação entre indivíduos que, por sua vez, depende da colaboração construída entre os membros da comunidade de aprendizado, seja ela online ou não;

- Compartilhamento: segundo Holanda (2010), é a ação ou efeito de 
compartilhar algo com alguém. Badcoock (2004) aponta problemas que fazem com que as pessoas evitem o compartilhamento. Segundo o autor, as pessoas não querem compartilhar o que sabem porque acreditam que conhecimento é um diferencial frente aos outros; o segundo correlaciona-se com o primeiro que é o fato de não haver confiança entre os indivíduos;

- Conversação: é a possibilidade de dois indivíduos, ou um grupo de indivíduos, estabelecerem comunicação direta (síncrona e/ou assíncrona) (Pereira, 2010b). Dourish e Bly (1992) analisaram a CMC (Comunicação síncrona e assíncrona Mediada por Computador) e avaliaram que a CMC consegue desenvolver uma consciência social colaborativa em relações, seja ela de trabalho ou informais;

- Grupo: segundo Holanda (2010), um grupo se refere ao número de pessoas ou de coisas que formam um todo;

- Objeto: artefato em torno do qual as interações sociais ocorrem (p. ex., ponto focal a partir do qual as discussões surgem, a conversa é mantida, a colaboração acontece) (Pereira et al., 2010);

- Relacionamento: algum tipo de ligação, laço social, entre dois ou mais indivíduos (Pereira et al., 2010);

- Propriedade: segundo Holanda (2010), é o direito pelo qual alguma coisa pertence a alguém.

\section{O Modelo de dados Proposto}

A Figura 1 ilustra o modelo MASSVA seguindo a notação do diagrama de classe da UML. Seus elementos para o contexto de aplicação relacionado à análise da VUX de um SS são os seguintes:

- SS: refere-se ao sistema, sendo o objeto de avaliação. É ele que fornece os dados, ou seja, as postagens;

- Postagem: trata-se da mensagem textual descrita pelo usuário durante o uso do SS em avaliação. A postagem pode ser dos tipos PRU e NPRU, definidos por Mendes (2015).

- Valor Humano: uma postagem PRU ou N-PRU expressa um ou mais valores humanos, afirmação descoberta quando a análise VUX foi feita em 2.000 postagens do Twitter, Oliveira et al. (2016). No modelo, um valor humano pode ser de dois tipos: valor Pessoal, o qual pode se relacionar com um ou mais valores pessoais e sociais; e valor social, o qual pode se relacionar com um ou mais valores pessoais e sociais.

- Relacionamento entre Postagem e Valor: a existência de valores humanos pessoais e sociais em PRU e em N-PRU deu origem à definição de dois elementos:

- VRU (Valor Relacionado ao Uso do Sistema), que é uma PRU que expressa valor. Por exemplo: a postagem PRU "Daqui a pouco tão mudando o nome do Twitter pra Orkut pq néh ... : $\rho$ " expressa o valor pessoal de emoção e afeto e os dois valores sociais de objeto e NRP;

o N-VRU (Valor Não Relacionado ao Uso do Sistema), que é uma Não- 
PRU que também expressa valor. Por exemplo: a postagem Não-PRU “@USER muito obrigado pra following! yeah brazil !!” expressa o valor pessoal de emoção e afeto e os sociais de objeto e conversação.

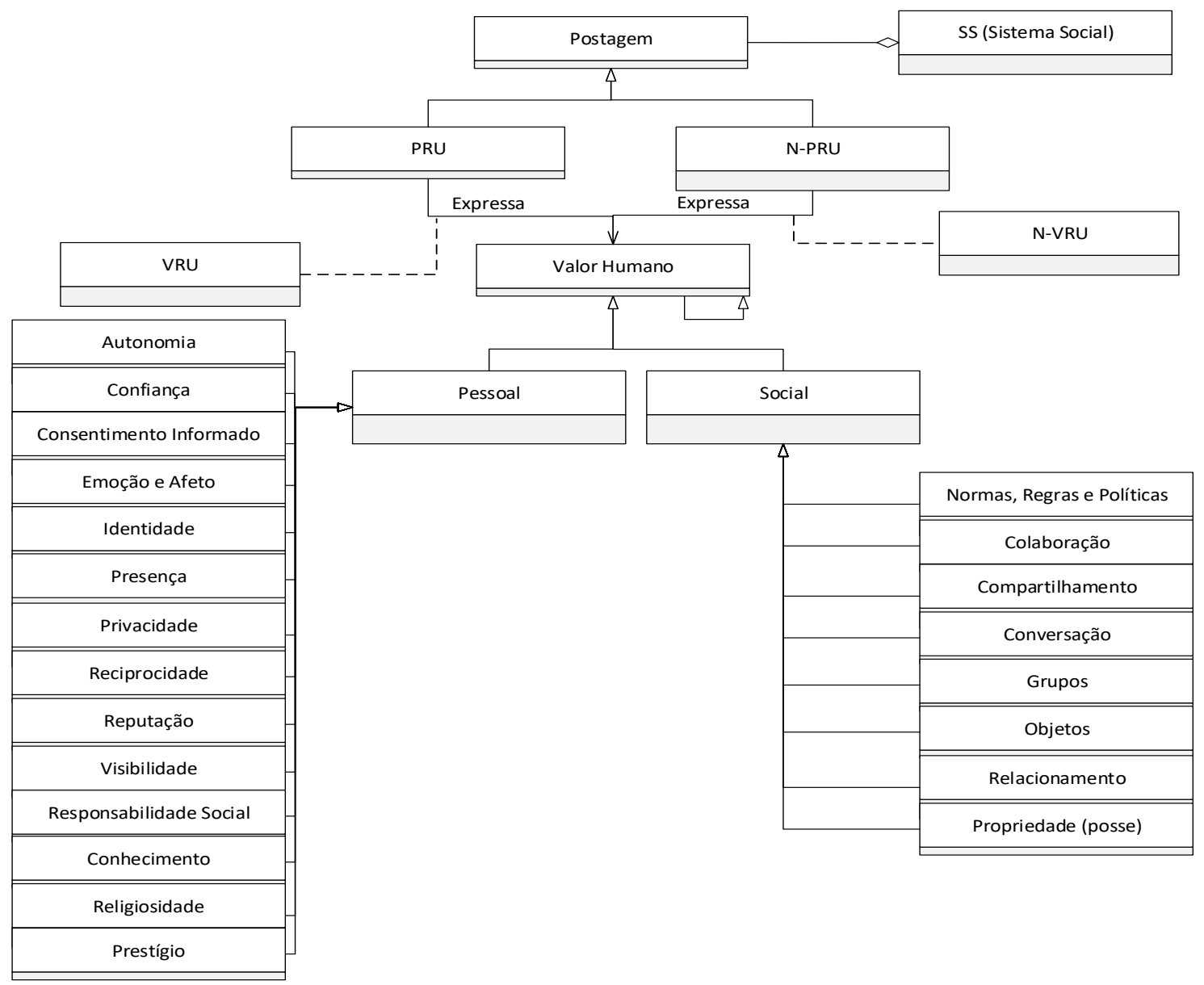

Figura 1. Modelo de dados MASSVA

\section{Aplicação do modelo de dados MASSVA}

Esta seção apresenta o uso do MASSVA num processo de classificação de postagens para um certo contexto resultando em uma formalização dos conceitos.

\subsection{Contexto de aplicação}

O Twitter foi o SS escolhido para a análise da VUX com três conjuntos de postagem, sendo eles: a primeira extração de postagens foi obtida a partir do banco de dados usando em (Mendes, 2015), consistindo de 1.000 postagens VRUs e outras 1.000 N-VRUs. Os resultados da análise da VUX de todas as postagens VRUs e N-VRUs foram apresentados em Oliveira et al. (2016); a segunda extração de postagens foi de 2.800 postagens, extraídas a partir do uso da ferramenta UUX-POSTs ${ }^{1}$, durante o mês de setembro/2016. Destas, 2.250 eram N-PRUs, dentre as quais, 550 eram postagens

\footnotetext{
${ }^{1}$ UUX-Posts: é uma ferramenta de extração e classificação de postagens relacionadas a Usabilidade e Experiência do Usuário (UX). Mendes (2015). Disponível em: http://200.129.62.41/luqs/analisador/v3/
} 
VRUs. As 550 postagens VRUs foram analisadas, quando dois valores pessoais eram identificados em uma mesma postagem, a postagem era repetida para facilitar $\mathrm{o}$ mapeamento dos problemas em qualidade em uso com os valores, resultando em 690 postagens a serem analisadas. Os resultados serão apresentados no tópico 5 deste artigo; e a terceira extração de 526 postagens, coletadas com a ferramenta UUX-POSTs em outubro/2016. Essa terceira extração foi utilizada para validação da classificação, descrita no tópico a seguir.

\subsection{Estratégia para classificação de valores nas postagens}

A classificação, entre valores pessoais e sociais, das 550 postagens PRUs da segunda extração foi realizada pelo pesquisador, autor principal deste artigo, sendo feita uma análise qualitativa e interpretativa considerando o conceito de cada valor (tópico 2.2). A terceira extração de postagens (526 postagens) foi fornecida a um especialista em análise da interação de SS para realizar uma classificação utilizando os mesmos conceitos. Houve um treinamento prévio para o especialista, que consistiu de: contextualização da importância da validação e passagem de conhecimento dos conceitos de cada valor. Enquanto isto, o pesquisador analisou os termos das postagens da segunda extração, já classificadas em cada valor, e elaborou uma formalização para definir, de forma gramatical, cada valor. A formalização de um valor é caracterizada por um conteúdo gramatical (P. ex., uma frase, palavra(s), ação(ões)), atuando como marcador utilizado para classificar cada postagem em um determinado valor.

Devido a problemas de espaço neste texto, destaca-se um exemplo de uma formalização, representada por "F", utilizando a teoria dos conjuntos. Para uma postagem VRU, representada por " $p$ ", expressando o valor pessoal autonomia, tem-se: $\mathrm{F}_{\mathrm{aut}}=\left\{\forall \mathrm{p} / \mathrm{E}_{1} * \mathrm{E}_{3}\right.$ ou $\left.\mathrm{E}_{2} * \mathrm{E}_{3}\right\}$, considerando " $\mathrm{E}$ " é um conteúdo gramatical pertencente a um conjunto, ou seja, onde $\left(E_{1} \in A\right),\left(E_{2} \in B\right)$ e $\left(E_{3} \in C\right)$, sendo $A=\{a /$ a é o desejo ou expectativa que aponta para a possibilidade ou impossibilidade de realizar algo em um $\mathrm{SS}\}$ ou seja, $\mathrm{A}=\{$ querer, desejar, ser, esperar, aguardar, $\ldots\} ; \mathrm{B}=\{\mathrm{b} / \mathrm{b}$ é a opinião positiva ou negativa sobre alguma ação do $\mathrm{SS}\}$, ou seja $\mathrm{B}=\{$ amei, odiei, perfeito, bonito,... $\}$ e $\mathrm{C}=\{\mathrm{c} / \mathrm{c}$ é uma ação $\}$, ou seja $\mathrm{C}=\{$ fazer, instalar, apagar, atualizar, colocar, mudar, modificar, editar, usar,...\} se $\left(\mathrm{A}^{*} \mathrm{C}\right) \neq \varnothing,(\mathrm{B} * \mathrm{C}) \neq \varnothing$.

Quando os dois finalizaram suas atividades, o pesquisador validou a formalização, verificando se a classificação, realizada pelo especialista, usando os conceitos dos valores, estava condizente com as formalizações, e realizou os ajustes quando necessários. Está fora do escopo deste trabalho, explicar os resultados alcançados com a comparação da classificação com a formalização, detalhes podem ser encontrados em Oliveira et al. (2016).

Porém é importante ressaltar que tal formalização ajudou o pesquisador a responder à questão desta pesquisa: "Quais valores pessoais e sociais são mais recorrentes e como estes podem ser úteis para avaliar a VUX dos SS? ". Ele passou de uma análise "ad-hoc" baseada na definição dos conceitos para uma análise sistemática dos elementos gramaticais. Ele utilizou 690 valores pessoais identificados nas 550 postagens coletadas na segunda amostra, e os resultados são apresentados na próxima seção. 


\section{Resultados}

Esta seção apresenta os resultados das análises VUX das postagens VRUs e NVRUs do Twitter, sendo eles: os valores e problemas de qualidade em uso mais recorrentes; as possibilidades de melhorias do sistema e os relacionamentos entre valores.

\subsection{Valores pessoais e sociais $\mathrm{X}$ Problemas mais recorrentes}

A Tabela 1 apresenta os valores mais expressados considerando o total de 690 VRUs. Dentre eles, a emoção e afeto tem 337 (48\%); autonomia 164 (23\%); confiança $95(14 \%)$, conhecimento $75(11 \%)$ e consentimento informado $19(3 \%)$. Os seguintes valores sociais (colaboração, grupo, relacionamento) e pessoais (identidade, presença, privacidade, reciprocidade, reputação, visibilidade) não foram representados por não terem sido classificados em nenhuma das postagens.

A Tabela também representa os problemas de qualidade em uso, por meio das medidas de UX. É importante descrever a que se referem as colunas "evidencia problema (Qtd) e evidencia problema (\%)". A primeira refere-se à quantidade de postagens VRUs, que evidenciaram problemas para um certo valor pessoal. Uma mesma postagem VRU pode evidenciar mais de um problema (p. ex., "Me dá uma raiva $o$ Twitter ficar mostrando que tem notificação, ai vc entra e não tem nada"). Tal exemplo foi classificado no valor pessoal autonomia e evidencia os problemas de usabilidade e de awareness. No caso da segunda, trata-se do percentual de postagens VRU, que evidenciaram problemas, para um certo valor pessoal. Por exemplo, o valor consentimento informado teve o percentual de $79 \%$ postagens que evidenciaram algum problema de qualidade em uso, percentual este calculado considerando 15 problemas em 19 postagens com esse valor. Apesar do percentual alto, a quantidade maior de problemas foi identificada no valor confiança (66).

Tabela 1. Valores pessoais que expressam problemas de qualidade em uso

\begin{tabular}{|c|c|c|c|c|c|c|c|c|c|c|c|c|c|c|c|}
\hline & \multirow[b]{2}{*}{ 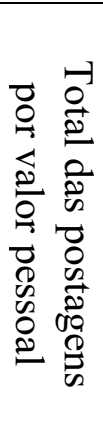 } & \multicolumn{5}{|c|}{ Valores Sociais } & \multicolumn{6}{|c|}{$\begin{array}{c}\text { Problemas de } \\
\text { qualidade em uso }\end{array}$} & \multirow[b]{2}{*}{ 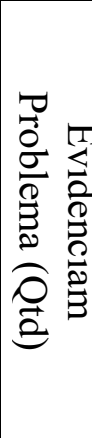 } & \multirow[b]{2}{*}{ 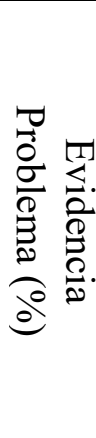 } \\
\hline & & & Z & 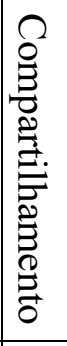 & 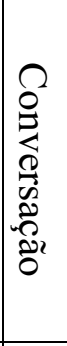 & $\frac{0}{0} \frac{0}{0}$. & 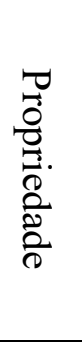 & 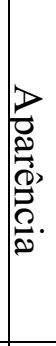 & 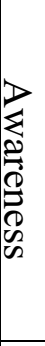 & 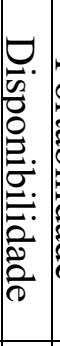 & 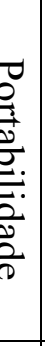 & 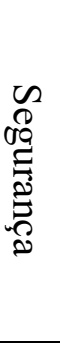 & 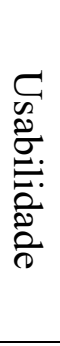 & & \\
\hline \multirow{5}{*}{ 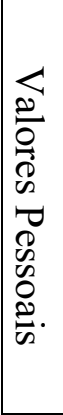 } & Autonomia & 164 & 24 & 2 & 8 & 164 & 118 & 1 & 1 & 0 & 1 & 0 & 11 & 13 & $8 \%$ \\
\hline & Confiança & 95 & 5 & 4 & 6 & 95 & 87 & 1 & 2 & 7 & \begin{tabular}{|l|}
3 \\
3 \\
\end{tabular} & 2 & 57 & 66 & $69 \%$ \\
\hline & $\begin{array}{l}\text { Consentimento } \\
\text { Informado (CI) }\end{array}$ & 19 & 0 & 0 & 2 & 19 & 15 & 0 & 2 & 0 & 0 & 2 & 13 & 15 & $79 \%$ \\
\hline & $\begin{array}{c}\text { Emoção e } \\
\text { Afeto }\end{array}$ & 337 & 37 & 18 & 32 & 337 & 134 & 1 & 3 & 0 & 0 & 2 & 37 & 40 & $12 \%$ \\
\hline & Conhecimento & 75 & 5 & 6 & 25 & 75 & 22 & 2 & 0 & 0 & 0 & 0 & 3 & 5 & $7 \%$ \\
\hline
\end{tabular}


Para exemplificar os problemas de qualidade em uso relacionados aos valores pessoais preparou-se a seguinte lista (descrita fielmente pelos usuários):

- Autonomia x Awareness: "Eu odeio meu Twitter ficar postando quem visualizou meu perfil. Que <palavrão>";

- Autonomia x Portabilidade: "só iOS que não tem como o twitter ficar como modo noturno??? pq essa < palavrão> não muda nada com a atualização";

- Confiança x Aparência: "atualizei meu twitter mais uma vez e até agora nao apareceu o modo noturno :)))))";

- Confiança x Awareness: "No meu Twitter ficar aparecendo toda hora que tem notificação, entro e não tem nada";

- Confiança x Disponibilidade: "nosfa to de saco cheio do twitter ficar parando";

- Confiança x Portabilidade: "Finalmente consegui fazer o Twitter entrar pelo celular de novo !!! Mas ainda tá bugado pra <palavrão > ;-;"

- Confiança x Segurança: "\{User\} só fiquei surpreso do twitter ter me mostrado a sua conta. hahaha";

- Consentimento Informado x Awareness: "Obrigada twitter, mais não quero saber quantas pessoas visualizaram meu perfil ?";

- Consentimento Informado x Segurança: "Nao sei como resolver isso do meu twitter ficar respostando sozinho";

- Consentimento Informado x Usabilidade: "se o twitter mudar a cor do icon ou da header, cabeças vão rolar";

- Emoção e Afeto x Aparência: "ate quando pro twitter deixar nossos icons com qualidade de novo";

- Emoção e Afeto x Awareness: "Agora Twitter dar pra ver a $<$ palavrão $>$ de dm visualizada, que triste";

- Emoção e Afeto x Segurança: "De alguma maneira fizeram meu perfil só Twitter seguir quase 400 sem a minha permissão. Tive que dar unfollow manualmente em todos eles.";

- Emoção e Afeto x Usabilidade: "nossa meu twitter ficar travando toda hora $q$ raiva";

- Conhecimento x Aparência: "o que dizer sobre o twitter ter tirado o modo noturno? Decepcionante";

- Conhecimento x Usabilidade: "N sei o pq do Twitter ficar apagando a foto com a frase q eu posto".

\subsection{Melhorias do SS}

Nesta sub-seção ilustram-se as possibilidades de melhorias. Os exemplos serão dados para as postagens N-VRUs, para ilustrar que a análise da VUX não se limita aos problemas de interação. Essas postagens são caracterizadas quando seu conteúdo não estiver relacionado ao uso do sistema, e sim a qualquer outro assunto (p. Ex., "tem gente q ta aqui a 3 anos, e tem 20 mil tweets, eu to aqui a 1 ano e pouco e já tenho 124 mil"). Por este motivo, elas não foram consideradas até agora. Ao analisar o elemento gramatical das postagens N-VRUs classificadas no valor prestígio e apresentadas na Tabela 2, foi percebido que, muito embora o usuário não falasse sobre o SS, tais frases poderiam apontar possíveis melhorias ao SS, como: "Falta pouco pra 15 mil tweets (:"; "Daqui a pouco eu chego nos 2000 tweets"; "Bem perto dos 800 followers! :D". 
Considerando tais exemplos, bem como outras postagens com conteúdo semelhante, como melhoria, seria interessante uma solução para a interface do SS Twitter, que pudesse informar a quantidade de Tweets (postagens) que o usuário já criou e uma outra solução para indicar quantos Followers (seguidores) cada usuário possui. As melhorias poderiam passar as informações necessárias variando os aspectos gráficos ou modalidades de interação, segundo o designer. Assim, cada usuário teria uma ideia da quantidade destas informações sem que precisasse perguntar a um outro usuário ou que este o informasse. A Tabela 2 apresenta mais exemplos, bem como a motivação e sugestões de melhorias a partir da análise das postagens N-VRUs.

Tabela 2. Sugestões de melhorias a partir da análise das postagens VRU

\begin{tabular}{|c|c|c|}
\hline Postagens & $\mathrm{Mo}$ & Sugestão de 1 \\
\hline "Falta pouco pra 15 mil tweets (:" & \multirow{4}{*}{$\begin{array}{l}\text { Possibilitar que } \\
\text { usuários tenham } \\
\text { uma noção de } \\
\text { quantos tweets cada } \\
\text { usuário tem sem } \\
\text { que precise ser } \\
\text { perguntado }\end{array}$} & \multirow{4}{*}{$\begin{array}{c}\text { Adicionar um elemento } \\
\text { gráfico que indique aos } \\
\text { usuários quantos tweets } \\
\text { ele já postou. Este } \\
\text { elemento poderia } \\
\text { trabalhar com cores, onde } \\
\text { cada cor representaria } \\
\text { uma quantidade mínima e } \\
\text { máxima. }\end{array}$} \\
\hline $\begin{array}{l}\text { "Quase atingindo } 1000 \text { tweets ... mais } \\
\text { eh muitoo pouco" }\end{array}$ & & \\
\hline "Falta pouco para os 4 mil tweets :P" & & \\
\hline $\begin{array}{l}\text { "tem gente q ta aqui a } 3 \text { anos, e tem } 20 \\
\text { mil tweets, eu to aqui a } 1 \text { ano e pouco } \\
\text { e já tenho } 124 \text { mil" }\end{array}$ & & \\
\hline "Bem perto dos 800 followers! :D" & \multirow{3}{*}{$\begin{array}{l}\text { Possibilitar que } \\
\text { usuários tenham } \\
\text { uma noção de } \\
\text { quantos followers } \\
\text { (seguidores) cada } \\
\text { usuário tem sem } \\
\text { que precise ser } \\
\text { perguntado }\end{array}$} & \multirow{3}{*}{$\begin{array}{l}\text { Adicionar um elemento } \\
\text { gráfico que indique aos } \\
\text { usuários quantos tweets } \\
\text { ele já postou. Este } \\
\text { elemento poderia } \\
\text { trabalhar com cores, onde } \\
\text { cada cor representaria } \\
\text { uma quantidade mínima e } \\
\text { máxima. }\end{array}$} \\
\hline $\begin{array}{l}\text { "quero } 100 \text { follower } \\
\text { até sexta" }\end{array}$ & & \\
\hline $\begin{array}{l}\text { "fcs se importam muito com followers" } \\
\text { desculpa, sou eu que tenho 5.000" }\end{array}$ & & \\
\hline
\end{tabular}

As análises realizadas nas postagens N-VRUs, as quais não possuam conteúdo relacionado ao uso do sistema, tiveram o intuito de verificar se estas podem colaborar para a melhoria de um SS. Falamos de melhoria porque não há uma reclamação por parte do usuário, tal como encontramos em postagens VRUs que, por sua vez, apontam uma solução para um possível problema apontado pelo usuário. O exemplo apontado na Tabela 2 aponta que é possível utilizar as postagens N-VRUs como fonte de informação para possíveis melhorias no SS.

\subsection{Relacionamento entre os valores pessoais e sociais}

Para investigar os relacionamentos entre valores pessoais e sociais, usou-se as três amostras mencionadas. Para simplificar a investigação e ilustrar o relacionamento, procurou-se identificar os valores pessoais que expressam um certo valor social. A Tabela 3 ilustra o percentual de postagens classificadas nos valores pessoais de autonomia e confiança que expressam também o valor social de propriedade. Considerando estas informações, analisou-se, de forma quantitativa, a seguinte suposição: obtendo-se a extração de, ao menos, duas postagens VRUs, classificadas nos 
valores pessoais autonomia ou confiança, pode-se ter também a expressão do valor social propriedade.

Tabela 3. Postagens de autonomia e confiança que expressam propriedade

\begin{tabular}{|l|c|c|c|c|c|c|}
\hline & Autonomia & Propriedade & $\%$ & Confiança & Propriedade & $\%$ \\
\hline $1^{0}$ Extração & 77 & 56 & 73 & 258 & 247 & 96 \\
\hline $2^{0}$ Extração & 164 & 118 & 73 & 95 & 87 & 92 \\
\hline $3^{0}$ Extração & 50 & 47 & 94 & 20 & 18 & 90 \\
\hline
\end{tabular}

O coeficiente de correlação de Pearson (r) foi utilizado para avaliar esta suposição. Com esse coeficiente, é possível obter o grau de relação linear entre duas variáveis quantitativas. Esse grau tem a seguinte variação $(-1 \leq r \leq 1)$ onde $r=1$ significa que duas variáveis são perfeitamente correlacionadas positivamente, ou seja, tem-se uma proporção de quantidade entre duas variáveis de $(1,1)$, enquanto $(0,8 \leq \mathrm{r} \leq 1)$ considera-se que há uma forte correlação entre duas variáveis quantitativas. Utilizando os valores da Tabela 3 e a fórmula de Pearson $\boldsymbol{r}=\frac{\sum(\boldsymbol{x}-\overline{\boldsymbol{X}})(\boldsymbol{y}-\overline{\boldsymbol{y}})}{\sqrt{\sum(\boldsymbol{x}-\overline{\boldsymbol{x}})^{2} \sum(\boldsymbol{y}-\bar{y})^{2}}}$, o coeficiente foi de $r=0,9937$ para o relacionamento de autonomia $X$ propriedade e $r=0,9999$ para $o$ relacionamento do valores confiança $\mathrm{X}$ propriedade.

O resultado do coeficiente de correlação de Pearson ajuda a sustentar a hipótese sobre a expressão destes valores pessoais de autonomia e confiança com o social de propriedade.

\section{Trabalhos Relacionados}

Nesta seção, mostramos que parte dos estudos do estado da arte que avaliam sistemas fazem uso de no máximo três valores:

Friedman (2006) trabalha com os valores de consentimento informado, privacidade e autonomia. Ele afirma que o consentimento informado fornece uma proteção extra para a privacidade quando este valor é utilizado em sites que coletam dados de usuários.

Donath (1998) afirma que o valor de identidade desempenha um papel chave em comunidades virtuais. O objetivo do autor é compreender como a identidade é estabelecida em uma comunidade on-line e as condições que dão origem à ambiguidade.

Lee, Antoniadis e Salamatian (2010) estudam o comportamento de usuários de comunidades sociais e de compartilhamento de conteúdo em termos de reciprocidade. Os autores acreditam que um usuário se depara com algum conteúdo, que considera importante para ele, então ele estabelece um sentimento de reciprocidade com quem o disponibilizou.

Bedi e Gove (2012) propõem um modelo de preservação dos usuários em SS baseando-se nos princípios de Hipócrates. Esses princípios são apontados por Agrawal et al. (2002), sendo eles: motivação específica, consentimento, conjunto mínimo, uso limitado, divulgação limitada, retenção limitada, exatidão, acesso e conformidade.

Troussas et al. (2013) afirmam ser importante saber como as pessoas se sentem 
sobre determinados temas os quais elas encontram nos SS. Ele propõe uma classificação destes sentimentos para o aprendizado de linguagem natural utilizando o algoritmo Naive Baÿes. O autor enfatiza que a identificação destes sentimentos é um processo complexo pelo fato dos sentimentos dos participantes de uma determinada discussão serem representados de várias maneiras.

Para Srivastava et al. (2008) a privacidade das informações dos usuários dos SS deve ser garantida e ser um dos principais objetivos dos desenvolvedores.

Neste artigo propomos um modelo de dados que avalia os SS utilizando vários valores pessoais e sociais. O objetivo é enriquecer as informações e transformá-las em conhecimento pelo fato da avaliação não estar direcionada a um ou outro valor pessoal ou social e sim a todos os valores que estejam expressados nas postagens do SS.

\section{Discussão}

Discutem-se as decisões e limitações da aplicação do modelo MASSVA, em termos dos seguintes requisitos usados para a classificação dos valores e análise VUX:

I) Tipos de análise: a escolha do tipo de análise da VUX de um SS dependerá primordialmente dos objetivos que se deseja alcançar. Os tipos podem ser: análise preditiva que, para Prates (2003), é realizada quando os avaliadores, ao analisarem os dados coletados, tentam prever que tipo de problemas os usuários enfrentarão; e a análise interpretativa, que é realizada quando os avaliadores procuram explicar os fenômenos que ocorreram durante esta interação. Esta proposta se encontra suportando apenas a análise interpretativa e, como tal, de difícil realização (ver próximo item);

II) Classificação manual e/ou automática: sabe-se que um processo de classificação manual é interpretativo. Então, uma mesma classificação pode ser feita de forma diferente pelos envolvidos, pois tem diferentes conhecimentos e podem apresentar diferentes pontos de vista. Para amenizar este tipo de problema, podem-se colocar vários indivíduos para avaliar e usar ferramentas de apoio a atividades colaborativas (para suportar técnicas de crowdsourcing, por exemplo). Nesta pesquisa, resolveu-se adotar uma estratégia para viabilizar uma classificação semi-automática, significando que parte do processo teve atividades manuais para definição, comparação e evolução da definição dos conceitos, e parte do processo de classificação (como a coleta, e análise quantitativa) foi automatizada;

III) Formalização dos conceitos como alternativa para amenizar o caráter subjetivo da classificação: para amenizar as incertezas de um processo subjetivo de interpretação dos conceitos sobre os valores, foi feita a formalização de cada um dos valores, dado um treinamento sobre os conceitos. O estabelecimento das formalizações ajuda a diferenciar cada valor e assim possibilitar o desenvolvimento de algoritmos de classificação das postagens de maneira automatizada. As formalizações poderão ser criadas a partir da análise de um conteúdo das postagens. O procedimento de classificação e validação deverá ocorrer apenas uma vez para cada SS que seja avaliado pelo modelo MASSVA já que o resultado da validação dos padrões será a automatização. Porém, sabe-se que não é possível uma formalização de classificação que seja absolutamente igual para um mesmo valor pessoal ou social em diferentes SS. Sabe-se, também, que qualquer trabalho de pesquisa evolui. Acredita-se numa classificação baseada na Colaboração entre os avaliadores; eles deverão validar a formalização (realizando experimentos de 
classificação com indivíduos não especialistas), (re) classificar quando necessário, evoluir os conceitos, e (re) definir padrões para apoiar uma classificação automática de todos os valores;

IV) Problemas para a qualidade em uso como dados do MASSVA. O modelo não possui os valores técnicos, tal como usabilidade, portabilidade, dentre outros, permitindo tão somente avaliar a expressão de valores em um SS, sem considerar a existência de problemas. Cabe ao avaliador definir o objetivo da análise da VUX de um $\mathrm{SS}$;

V) Perfil do avaliador: segundo Preece et al. (1994), o avaliador poderá ter conhecimentos: a) de domínio, onde o avaliador deverá estar familiarizado com o SS que está sendo analisado com relação às suas principais funções e contextos de uso; b) de conhecimento sobre interface de usuário, no qual o avaliador deverá ter conhecimento sobre os principais aspectos de interação, terminologias, heurísticas e problemas que afetam a qualidade em uso; e c) o conhecimento estatístico, no qual o avaliador deverá ter conhecimento de várias técnicas estatísticas de forma a extrair conhecimentos a partir das informações obtidas. Essa estratégia deve ser seguida por qualquer indivíduo interessado pelo MASSVA (que pode ser pesquisador e aluno da área de IHC - especialista ou não).

VI) Ciclo de avaliação: a discussão sobre este item diz respeito ao momento que um SS poderá ser avaliado quanto à VUX sob a aplicação do modelo MASSVA. Foram considerados dois tipos: avaliações formativas que, segundo Preece et al. (1994) são aquelas feitas durante o processo de design, ou seja, antes do sistema estar terminado; avaliação somativa que, segundo Garau (2003), é realizada sobre o artefato final, ou seja, do sistema já em funcionamento.

\section{Conclusão}

O principal objeto da pesquisa apresentado neste artigo é o modelo de dados MASSVA, o qual possui elementos que ajudam na análise da VUX. Resultados foram apresentados neste artigo e, muito embora representem parte do total de resultados/análises obtidas na pesquisa, corroboraram para demonstrar que o MASSVA ajuda a direcionar a avaliação de problemas de qualidade em uso de SS. Primeiro porque, a relação entre os valores pessoais e sociais permitiram a definição de um formalismo sobre os conceitos. Depois, existe a relação entre os valores e as postagens relacionadas ao uso do SS, embora tenham aquelas que não estão relacionadas ao uso do SS. Descobriu-se que por meio dessas últimas, também é possível identificar possibilidades de melhorias no conteúdo e/ou na interface do SS. Verificou-se, ainda, que existem valores pessoais e sociais difíceis de serem identificados nas postagens, enquanto existem valores sociais que se encontram de forma mais frequente em certos valores pessoais.

Como trabalhos futuros, destaca-se: desenvolver algoritmos de classificação automatizada para cada um dos valores; definir uma metodologia de aplicação do modelo de dados MASSVA considerando as características apresentadas neste artigo; e aplicar o modelo de dados MASSVA, a partir de uma futura proposta de metodologia de aplicação deste modelo, em outro SS. 


\section{Referências}

Abu-Nimeh, S. ; Chen, T. ; Alzubi, O. (2011) "Malicious and Spam Posts in Online Social Networks". Em IEEE Computer Society, P 23-28.

Arevalo Velosa, Z.; Arcelio, H.F.; Carlos, C.I. (2010). "Social responsability of science and engineering”. In ANDESCON, P 1-6.

Bedi, R.; Gove, N.R. (2012) "Application of Hippocratic principles for privacy preservation in social network". Em (WICT), P 95-101.

Bedford, E., 1957. "Emotions," Proceedings of the Aristotelian Society, 57: 281-304.

Beiming Sun ; Dept. of Comput. (2014) "Analyzing sentimental influence of posts on social networks”, Hong Kong Polytech. Univ., Hong Kong, China. P 546-551.

Bolea, Speranta Cecilia; Romanian Academy. (2015) "Vocabulary, synonyms and sentiments of hazard-related posts on social networks". Em SpeD. P 14-17.

Donath, J. S. (1999) "Identity and Deception in the Virtual Community". Em: KOLLOCK Peter. e Marc Smith. New York: Routledge.

Dron, J., (2007) "Designing the undisignable: social software and control". Educational Technology \& Society. 10(3). P 60-71.

Garau M., (2003) "The impact of avatar fidelity on social interaction in virtual environments,". University College London.

Hogg, M.; Abrams D., 1988. Social Identications: A Social Psychology of Intergroup Relations and Group Processes. London: Routledge.

Holanda, Aurelio Buarque. Dicionário Aurélio da Língua Portuguesa. 5 th edição, 2010;

Japiassu, H. (1977). "Introdução ao pensamento epistemológico". 2. ed. Rio de Janeiro: F. Alves.

Kenny, A., 1963. Action, Emotion and Will, London; New York: Routledge and Kegan Paul; Humanities Press.

Klamma, R., Chatti, M., Duval, E., Hummel, H., Thora, E. (2007). "Social Software for Life-long Learning”. Educational Technology \& Society, Vol 10. pp.72-83.

Lee J. G.; Antoniadis, P.; Salamatian, K. (2010). "Faving Reciprocity in Content Sharing Communities: A Comparative Analysis of Flickr and Twitter". In ASONAM, P 136- 143.

Mayer, R. C., Davis, J. H. \& Schoorman, F. D. (1995) "An Integrative Model of Organizational Trust,” Academy of Management Review (20), pp. 709-734.

McLoughlin, C. and Lee, M (2007). "Social software and participatory learning: Pedagogical choices with technology affordances in the Web 2.0", Em: Ascilite Singapore, pp.664-675.

Mendes, M. (2015). "MALTU - Um modelo para avaliação da interação em sistemas sociais a partir da linguagem textual do usuário". Tese (doutorado). Universidade Federal do Ceará, Programa de Pós-Graduação em Ciência da Computação, Fortaleza, 2015. 
Morville, P. (2004) "User Experience Design".

Oliveira, D. C., Furtado, E. S., Mendes, M. S., (2016). "Do Users Express Values During Use of Social Systems? A Classification of Their Postings in Personal, Social and Technical Values". In 8th International Conference, SCSM 2016, Held as Part of HCI International. Volume 9742. P 130-140.

Pereira R., M. Cecília C. Baranauskas. (2010) "Softwares sociais: uma visão orientada a valores". In IHC, p. 149-158.

Pereira, R.; de Miranda, L.C.; Baranauskas, M.C.C.; Piccolo, L.S.G.; Almeida, L.D.A.; Dos Reis, J.C. Interaction design of social software: Clarifying requirements through a culturally aware artifact. Information Society (i-Society), P. 293 - 298, 2011.

Sabourin E., MARCEL MAUSS: Da dádiva à questão da reciprocidade. Revista Brasileira de Ciências Sociais, Vol.23, 2008.

Shirky, C. "A group is its own worst enemy: Social structure in social software". O' Reilly Emerging Technology conference.CA.Em http:/www.shi rky.com/ writings/group_enemy.html, 2005. Acesso em Dezembro/2015.

Schoeman, F., (1992). "Privacy and Social Freedom". Cambridge: Cambridge University Press.

Smith, G. Tagging (2008) "People-Powered Metadata for the Social Web". New Riders, Berkley.

Sorenson, O., R. Jan, Rivkin and F. Lee;(2006). "Complexity, networks and knowledge flow”,.Research Policy, vol. 35, P 994-1017.

Srivastava J.; Ahmad M.A.; Pathak N.; Hsu D. K. W.; (2008) "Data mining based social network analysis from online bahavior". Em (SDM);

The Belmont Report (1978): Ethical Principles and Guidelines for the Protection of Human Subjects of Research.

Troussas, C.; Virvou, M.; et all. (2013) "Sentiment analysis of Facebook statuses using Naive Bayes classifier for language Learning". Em (IISA). P 1-6.

Tsai, I.-C., Kim, B., Liu, P., Goggins S.P., Kumalasari, C. \& Laffey, J.M. (2008) "Building a Model Explaining the Social Nature of Online Learning". Educational Technology \& Society. 11(3). P 198-215.

Wang B., Jiting Yang, Hongpei Liu. (2010) "Understanding the Mechanism of Social Network in the Knowledge Transfer Process". Em PICMET, P.1-6.

Yang, S. J. H., I. Y. L. Chen, J. Huang and T. Fan, (2007) "Social network supports in knowledge sharing", Second International Conference on Innovative Computing, Information and Control, ICICIC. 